\title{
Formation and modulation of nanotubular assemblies of oligourea foldamers in aqueous conditions using alcohol additives
}

Sung Hyun Yoo,+[a] Gavin W. Collie,+[b] Laura Mauran,[a,c] and Gilles Guichard*[a]

\author{
[a] Dr. S. H. Yoo, Dr. L. Mauran, Dr. G. Guichard
}

Univ. Bordeaux, CNRS, Bordeaux INP, CBMN, UMR 5248

Institut Européen de Chimie et Biologie

2 rue Robert Escarpit, 33607, Pessac, France

E-mail: g.guichard@iecb.u-bordeaux.fr

[b] Dr. G. W. Collie

Discovery Sciences, R\&D

AstraZeneca, Cambridge, UK

[c] Dr. L. Mauran

UREKA Pharma SA,

2 rue Robert Escarpit, 33607, Pessac, France

[+] These authors contributed equally to this work.

Supporting information for this article is given via a link at the end of the document.

\begin{abstract}
There is considerable interest in the rational design of controllable, bioinspired supramolecular systems as a potential means to create new biocompatible and functional materials able to mimic and build upon the characteristics of natural biopolymers. Here, we report the alcoholcontrolled aqueous self-assembly of an amphiphilic helical oligourea foldamer (artificial folded oligomer) into a diverse array of tubular fibril architectures. Electron microscopy studies provide details of the morphological evolution of the foldamer nanostructures from protofibrils to fibers, with high resolution X-ray crystal structures providing an atomic-scale view of these assemblies, and solution studies indicating the assembly and morphology to be affected by alcohol polarity and concentration. Overall, the results we report here highlight oligourea foldamers as suitable building blocks for the formation of a diverse range of tubular morphologies in a controllable manner.
\end{abstract}




\section{Introduction}

Naturally abundant one-dimensional (1D) self-assembled architectures such as collagen, fibrin and microtubules are essential in biological systems, playing diverse roles, for instance, in cell adhesion and cell growth.[1-3] Taken as inspiration, significant efforts have been devoted towards the fabrication of novel biomimetic 1D materials based on natural polymers using a bottom-up approach for applications in biotechnology and synthetic biology.[4-7] Building blocks with proteinaceous side chains have attracted particular interest, in part due to the diverse chemical functionalities provided by the 20 naturally occurring amino acids and the subsequent diversity of architectures formed from polymers of these residues. For example, peptide amphiphiles, dipeptides and coiled-coil peptides have been reported to form 1D architectures such as fibers and nanotubes with controllable morphologies and functions.[8-15]

Alongside natural biopolymers, sequence-specific synthetic molecules mimicking the secondary structures of biopolymers, i.e. foldamers,[16-19] have recently emerged as building blocks for the creation of biomimetic supramolecular architectures. The combination of sequence control and folding fidelity by enabling precise positioning of polar and hydrophobic side chains at the surface of foldamer scaffolds, together with the exploitation of a wider range of monomeric units[20-24] make foldamers highly suitable systems for the design of complex and atomically precise nanostructures.[25-35] For instance, globally amphiphilic helical foldamers bearing proteinaceous side-chains have been shown to self-assemble into nanofibers via hydrophobic attraction in aqueous media.[31,32] In addition, the physical and chemical dissimilarity (e.g. folding rules and proteolysis resistance) between foldamers and natural biopolymers suggests that foldamer-based supramolecular systems may exhibit properties that could differ fundamentally from natural systems in terms of shapes and functions. However, the precise construction of foldamer-based 1D nanostructures requires a comprehensive understanding of the self-assembling properties of each foldamer backbone of interest. In particular, high resolution structural elucidation (e.g. by NMR or Xray crystallography) as well as extensive sequence engineering and careful examination of the role of environment factors (e.g. pH, temperature and additives) is needed. In this respect, the use of additives provides an appealing approach to study and modulate the aqueous self-assembly process of foldamers. Indeed, the control of self-assembling properties of natural biopolymers using small molecule additives has been studied in detail,[6],[36-38] while few examples have been reported for foldamer building blocks.[33,39]

Here, we describe how the self-assembling properties of an amphiphilic helical oligourea foldamer and the resulting tubular nanostructures can be tuned using alcohols as additives. High-resolution crystal structures reveal the foldamer to self-assemble into two distinct tubular structures with water-filled hydrophilic channels, decreasing in diameter with increasing alcohol concentration. Extensive studies by transmission electron microscopy (TEM) also revealed the foldamer to selfassemble into a diverse array of fibrillar structures in an alcohol-dependent manner. These structural studies were supported by circular dichroism (CD) data indicating a clear effect of alcohol concentration and polarity on the self-assembling behaviour of the oligourea foldamer.

\section{Results and Discussion}

\section{Amphiphilic helical building block design and aqueous self-assembly}

Previously, we have reported a water-soluble amphiphilic helical oligourea foldamer, termed H2 (Figure 1a-c), which was shown by a variety of techniques to self-assemble into an extended 
supramolecular tubule structure in aqueous conditions.[26] The self-assembly of $\mathrm{H} 2$ is driven by the global amphiphilicity of this 10-mer oligomer: the six uncharged residues (four leucine-, one prolineand one alanine-type urea residue) are clustered at the $a, c$ and e positions of the pentad repeat, with four charged residues (two glutamate- and two lysine-type urea residues) clustered at the $b$ and $\mathrm{d}$ positions of the pentad repeat (Figure $1 \mathrm{~b}$ ). By this sequence design, a continuous hydrophobic face is created on the helix's lateral surface, while a continuous hydrophilic region is formed on the opposite side, thus creating a globally amphiphilic structure (Figure 1c). This oligourea was initially synthesized using a solution condensation approach,[26] however, to explore the effect of sequence variations and accelerate the synthesis of analogues we decided to move to solid-phase synthesis. Towards this end, we developed a stepwise protocol on solid support, starting from Rink amide resin and using microwave assistance (full details can be found in the Supporting Information). As a consequence of the attachment to solid support, oligomers synthesized in this manner terminate with a monosubstituted urea and lack the methyl group of the C-terminal urea of $\mathrm{H} 2$ synthesised in solution (Figure 1a). In order to assess the effect of this C-terminal modification on the selfassembling properties of $\mathrm{H} 2$-related sequences, we first characterized $\mathrm{H}^{\prime}$, the direct analogue of $\mathrm{H} 2$ lacking the terminal methyl group. Variable-concentration circular dichroism (CD) analysis of $\mathrm{H2}^{\prime}$ in both buffered conditions at $\mathrm{pH} 7.4$ and in water showed that the molar residual ellipticity (MRE) at $202 \mathrm{~nm}$ (MRE202), the signature of helical oligourea foldamers in solution, increased as $\mathrm{H}^{\prime}{ }^{\prime}$ concentration increased (Figure $1 \mathrm{~d}$ and Figure S5). This stabilization effect upon increasing H2' concentration under both conditions is supportive of the existence of supramolecular assembly formation, and is in-line with the equivalent analysis of $\mathrm{H} 2$.[26] Negative-stained transmission electron microscopy (TEM) revealed the morphology of $\mathrm{H} 2^{\prime}$ supramolecular structure from a buffered system as protofibrils with diameters of $7.8 \pm 0.89 \mathrm{~nm}$ and lengths of $50 \pm 20 \mathrm{~nm}$ (Figure 1eg), again, in-line with equivalent analysis of $\mathrm{H} 2$ reported previously.[26] 

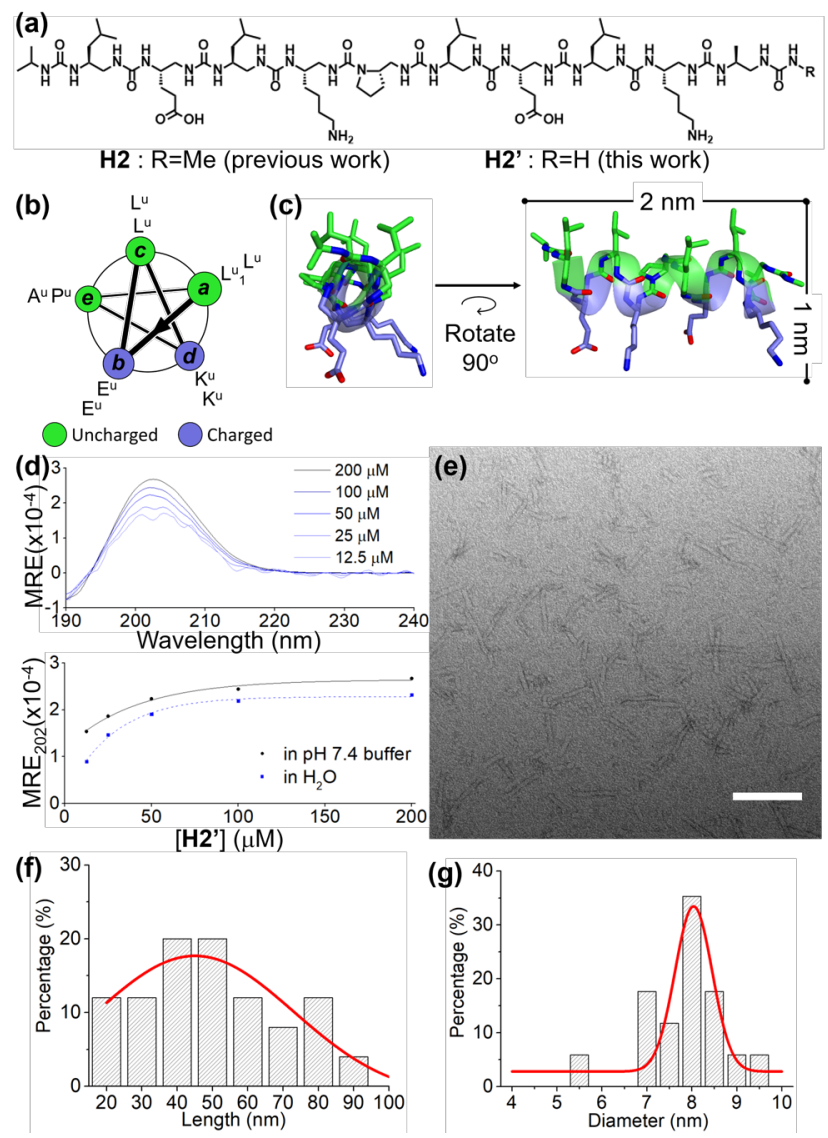

Figure 1. Amphiphilic helical oligourea foldamer $\mathrm{H}^{\prime}$ and its self-assembling behaviour in aqueous conditions. (a) Chemical structure of $\mathrm{H} 2[26]$ and $\mathrm{H}^{\prime}$ '. (b) Pentad repeat representation and (c) representation of the helical conformation in the crystal structure of $\mathrm{H} 2$. Superscript ' $\mathrm{u}$ ' represents urea residue. Uncharged and charged residues are coloured green and blue, respectively. (d) Variable-concentration CD analysis of H2' in 20 mM sodium phosphate ( $\mathrm{pH}$ 7.4) (top) and plot of H2' concentration versus MRE202 (molar residual ellipticity (deg $\mathrm{cm} 2$ dmol-1 residue-1) at $202 \mathrm{~nm}$ ) (bottom) in $20 \mathrm{mM}$ sodium phosphate buffer at pH 7.4 (black) and in water (blue). Trend lines for MRE202 are shown to guide the eye only and are not fit to a mathematical model. (e) Negative-stained TEM image of H2' from a solution composed of $100 \mu \mathrm{M} \mathrm{H2}$ ' plus $25 \mathrm{mM}$ sodium HEPES buffer (pH 7.4), incubated for 3 days at room temperature before analysis, revealing the presence of protofibril structures. Scale bar: $100 \mathrm{~nm}$. (f-g) Histogram profiles of (f) length and (g) diameter of the H2' protofibrils shown in (e). The red guide lines represent Gaussian distributions.

\section{Polymorphic crystal structures of $\mathrm{H2}^{\prime}$}

In order to further confirm that $\mathrm{H}^{\prime}$ ' was self-assembling into tubular structures similar to those formed from $\mathrm{H} 2$, we solved a crystal structure of $\mathrm{H}^{\prime}$ ' by $\mathrm{X}$-ray diffraction analysis of crystals grown using standard aqueous crystallisation techniques (hanging drop vapour diffusion). A crystal structure of $\mathrm{H}^{\prime}$ ' was determined from a crystal grown from a crystallisation solution composed of $0.2 \mathrm{M}$ sodium citrate, $100 \mathrm{mM}$ sodium HEPES buffered at $\mathrm{pH} 7.5$ and $20 \%(\mathrm{v} / \mathrm{v})$ isopropyl alcohol (IPA). This structure, belonging to space group P61 and refined to $1.21 \AA$, is isomorphous with that determined for $\mathrm{H} 2$ previously,[26] revealing the oligourea to form a canonical right-handed oligourea helix, which pack together to form a channel-type nanostructusacomposed of a double-stranded superhelix with a charged, water-filled interior pore (Figure 2a and Figure S7-S9). This structure therefore confirms that the C-terminal modification of $\mathrm{H2}^{\prime}$ (relative to $\mathrm{H} 2$ ) does not negatively affect the ability of this foldamer to self-assemble into a channel-type structure in the solid-state. 

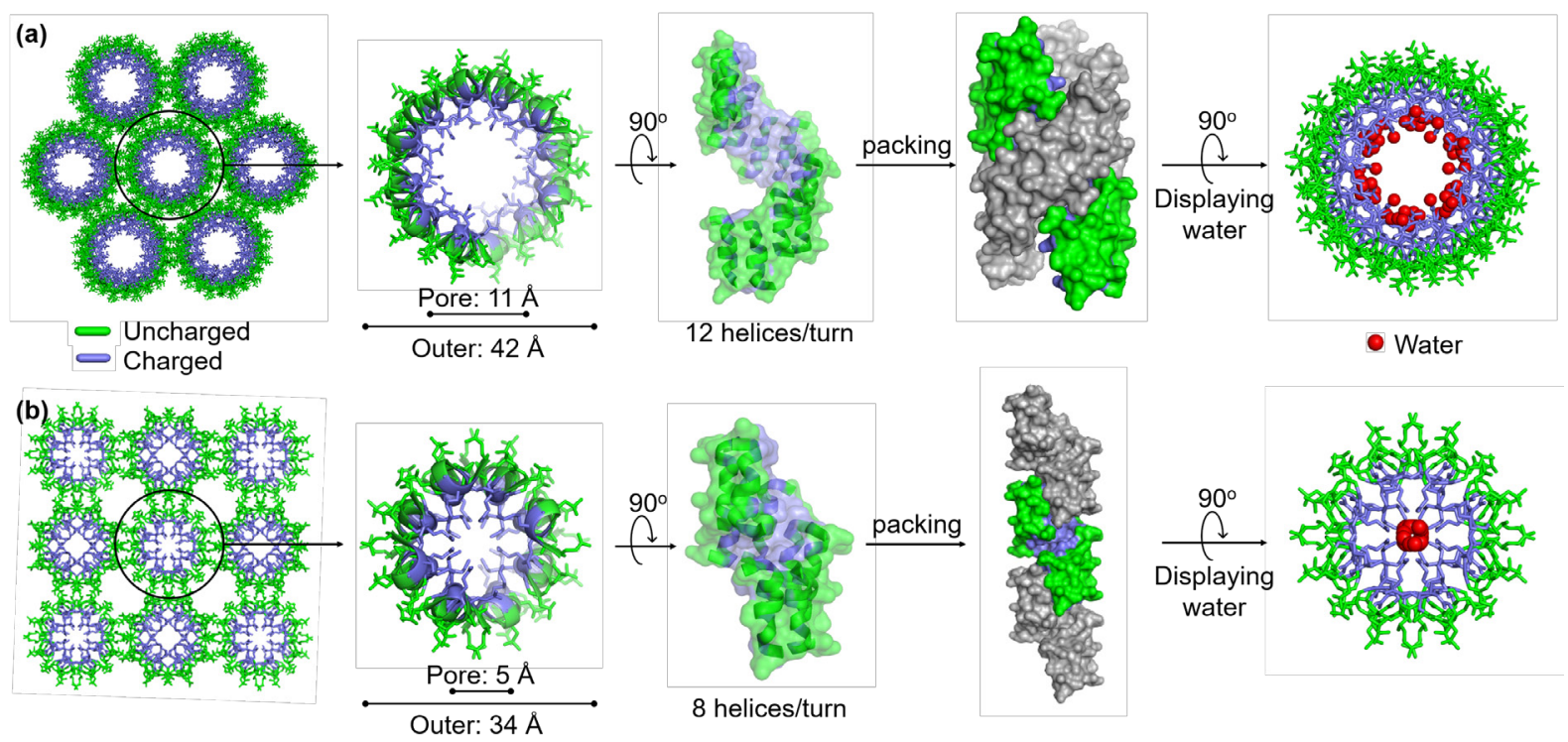

Figure 2. Crystal structures of $\mathrm{H}^{\prime}$ ' determined from crystals grown in the presence of (a) $20 \%$ IPA and (b) $35 \%$ IPA.[40] Uncharged residues and charged residues are depicted in green and blue, respectively. Water molecules are omitted for clarity except in the right panels depicting the water structure of the channels (in this panel some waters have been omitted for clarity, with the full water network shown in Figure S7). Pore and outer diameter measurements are provided with respect to the van der Waals radii (pore diameters were calculated using CAVER[41]). Superhelix units (middle-right) in (a) are coloured separately. Model description from left to right: stick, stick + helix drawing, surface + helix drawing, surface and stick + sphere (water).

In addition to this crystal structure, to our surprise, a second crystal structure was determined for $\mathrm{H} 2^{\prime}$, this time in space group $\mathrm{P} 4122$, from crystallisation conditions identical to those described for the P61 crystal form yet with a higher IPA concentration of 35\%.[40] This second crystal form (in space group $\mathrm{P} 4122$ ) also revealed $\mathrm{H} 2$ ' to fold into canonical oligourea helices, differing marginally to those observed in the P61 crystal form in that the C-termini of the helices in the P4122 crystal form are slightly unfolded (Figure S8-S9). Interestingly, the $\mathrm{H}_{2}$ ' helices of the P4122 crystal form also pack to construct a channel-type nanostructure with a highly charged, water-filled internal pore lined with carboxylic acids and amines from the Gluu and Lysu residues, respectively, and with a hydrophobic outer surface composed of the hydrophobic Leuu, Alau and Prou residues (Figure 2b and Figure S7). The oligourea helices pack side-by-side in an anti-parallel yet slightly laterally offset arrangement, thereby forming right-handed superhelices, similar to the inter-helical packing seen in the H2' P61 crystal form. However, despite sharing some similarity in inter-helical packing, there are striking differences in the overall quaternary arrangements seen in the two H2' crystal forms. In the P61 crystal form (grown from crystallisation conditions containing 20\% IPA), two superhelical strands of $\mathrm{H} 2^{\prime}$ (each of which contains twelve $\mathrm{H} 2$ ' helices per turn) intertwine to create a double-helical structure with a pore diameter of $11 \AA$ and an outer diameter of $42 \AA$ (measurements are provided with respect to the van der Waals radii). In contrast to this, $\mathrm{H2}$ ' helices of the P4122 crystal (grown from crystallisation conditions containing $35 \%$ IPA) form a single superhelical strand with just eight $\mathrm{H} 2$ ' helices per turn. With fewer superhelical strands and fewer $\mathrm{H} 2$ ' helices per superhelical turn, the channel-type structure in the P4122 crystal form has a smaller overall diameter than the P61 crystal form, with an interior pore diameter of $5 \AA$ and outer diameter of $34 \AA$.

\section{Alcohol-controlled fibrous growth of H2'}


The crystal structures described above revealed $\mathrm{H}_{2}$ ' to be capable of forming diverse channel-type structures, at least in the solid state. Furthermore, that the crystallisation conditions from which the two different crystal forms arose differ only in IPA concentration suggested that alcohol content may be driving the divergent assemblies. Indeed, analysis of the charged surface areas of the internal pores indicates clearly that the P4122 crystal form has far less charged surface exposed than the P61 crystal form,[42] consistent with solvent playing a key role in the assembly process.
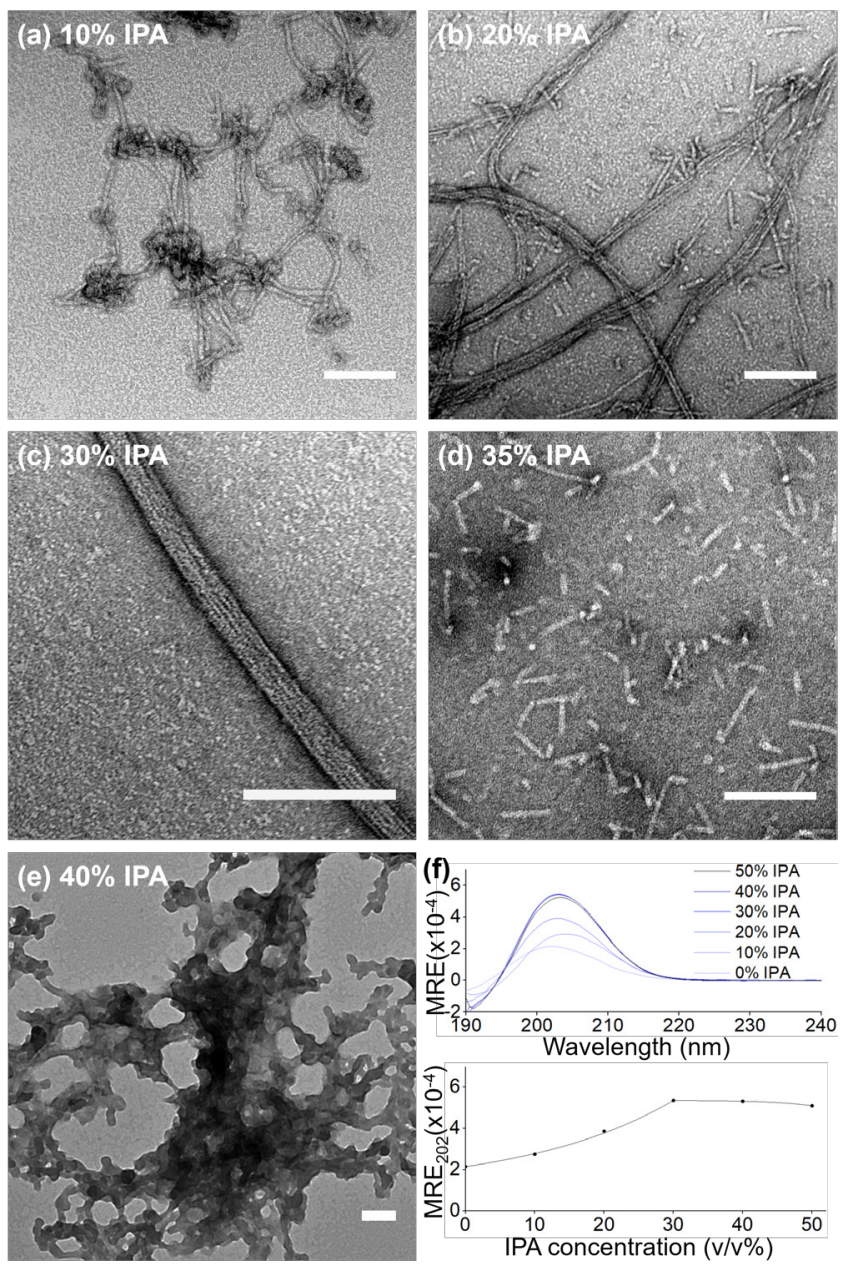

Figure 3. IPA controlled fibrous growth of H2'. (a-e) TEM images of $\mathrm{H} 2^{\prime}$ from solutions composed of $100 \mu \mathrm{M}$ H2', 25 mM sodium HEPES (pH 7.4) plus (a) 10\%, (b) 20\%, (c) 30\%, (d) $35 \%$ or (e) $40 \%$ (v/v) IPA, revealing a diverse array of nanostructures. Scale bars: $100 \mathrm{~nm}$. (f) CD analysis of H2' in $20 \mathrm{mM}$ sodium phosphate buffer $(\mathrm{pH} 7.4)$ in the presence of varying IPA concentrations after a 5 minute incubation period at room temperature (top) and plot of IPA concentration versus MRE202 (bottom). Trend line for MRE202 is shown to guide the eye only.

We thus sought to investigate the role that IPA, and alcohol in general, may be playing in $\mathrm{H}^{2}$ ' selfassembly using imaging and solution techniques. Analysis by TEM of $\mathrm{H}^{\prime}$ ' from aqueous solutions with differing IPA concentration after 3 days of aging time revealed $\mathrm{H}^{\prime}$ to form distinct and diverse fibril structures in response to alcohol concentration (Figure 3a-e and Figure S10). In the presence of $10 \%$ IPA, fibrils with lengths longer than $100 \mathrm{~nm}$ were observed, while their diameters remained consistent at around $7 \mathrm{~nm}$, similar to the dimensions of the protofibrils observed in the absence of IPA. At 20\% IPA, discrete fibrils, again with consistent diameters of around $7 \mathrm{~nm}$, were observed. In addition to these structures, fibril bundles (i.e. fibers), with lengths in the micron range and widths of around $20 \mathrm{~nm}$ were also observed. At $30 \%$ IPA, these fibril bundles are the predominant structural species observed by TEM, with the discrete fibrils essentially not observed. At 35\% IPA, the fibril 
bundles cannot be observed, and appear to be replaced by discrete fibrils with lengths shorter than $100 \mathrm{~nm}$. At 40\% IPA, no discernible ordered quaternary structures are visible, with only amorphous non-fibrillar aggregates observed. Overall, as the concentration of IPA is increased to $30 \%$, significant longitudinal growth (from lengths less than $100 \mathrm{~nm}$ to lengths in the $\mu \mathrm{m}$ scale; from protofibril to fibril) followed by fibril bundle (i.e. fiber) formation is observed, while the diameter (ca. $7 \mathrm{~nm}$ ) of each discrete fibril remains constant (Figure S10). Above this optimal IPA concentration for fibril formation, fibrous growth of $\mathrm{H} 2^{\prime}$ is suppressed, along with the induction of amorphous aggregation.

It is notable that both TEM and X-ray crystallography show clearly that: 1) H2' self-assembles into continuous tubular (or fibrillar) structures in the solid state, and 2) the morphology of the tubular (or fibrillar) assemblies changes in response to the concentration of alcohol (IPA) present. In addition we note that, although a direct comparison between the structures observed crystallographically with those observed by TEM is not possible, comparison of the dimensions nevertheless reveals the fibrils observed by TEM to be around $3 \mathrm{~nm}$ larger than the channels observed crystallographically. As the diameter of a single $\mathrm{H}^{\prime}$ ' helix is around $1 \mathrm{~nm}$, it is possible that the enlarged fibril diameter observed by TEM (relative to the crystallographic data) may be a result of a second layer of $\mathrm{H} 2^{\prime}$ helices packing against the hydrophobic outer surfaces of the tubular structure.

We next turned to solution studies using $C D$ (Figure $3 f$ ) in order to investigate further the effect of IPA on the self-assembling properties of $\mathrm{H}^{\prime}$ ', particularly the fibrils observed by TEM (Figure 3a-d). CD-monitored titration of IPA into an aqueous solution of $\mathrm{H}^{\prime}$ ' revealed an increase in helicity (as measured by MRE202) up to a concentration of 30\% IPA, beyond which the helicity plateaus (Figure 3f, bottom panel). Although MRE202 only indirectly measures quaternary structure (via the stabilising effect that quaternary interactions can exert on individual helices), these CD data are nevertheless highly consistent with the TEM analysis, which showed increasing quaternary structures from 0 to 30\% IPA (from short protofibrils to fibril bundles with increasing length and size), followed by a loss of discernible ordered assemblies at 40\% IPA (Figure 3e). Although the non-polar environment provided by increasing the concentration of IPA reinforces polar interactions and thus contributes to greater oligourea helix stabilization, the finding that fibrils are formed at an optimal concentration of IPA indicates the need for both polar and hydrophobic interactions to be strong enough.

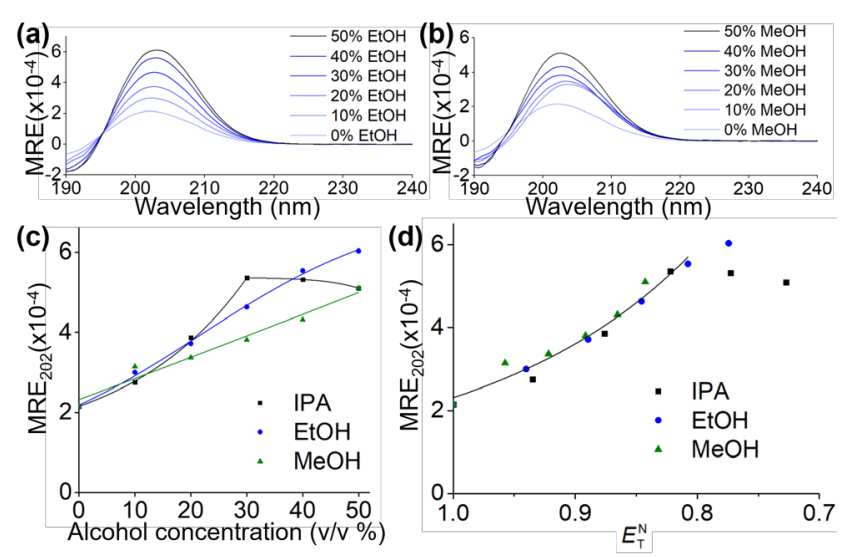

Figure 4. (a, b) $\mathrm{CD}$ analysis of $\mathrm{H}^{\prime}$ ' in $20 \mathrm{mM}$ sodium phosphate buffer (pH 7.4) with varying concentrations of (a) $\mathrm{EtOH}$ or (b) MeOH. (c) Plot of alcohol concentration versus MRE202 of H2' (in 20 mM sodium phosphate buffer, $\mathrm{pH}$ 7.4) in the presence of different alcohols (IPA (black), EtOH (blue) and $\mathrm{MeOH}$ (green)). (d) Equivalent plot to (c) but with the $x$ axis representing solvent polarity, (the normalized empirical parameter of solvent polarity) in 
place of alcohol concentration. values for alcohol-water binary solutions (10\%, 20\%, 30\%, 40\% and 50\% (v/v) alcohol in water) were deduced from reference [44]. Trend lines for MRE202 are shown to guide the eye only.
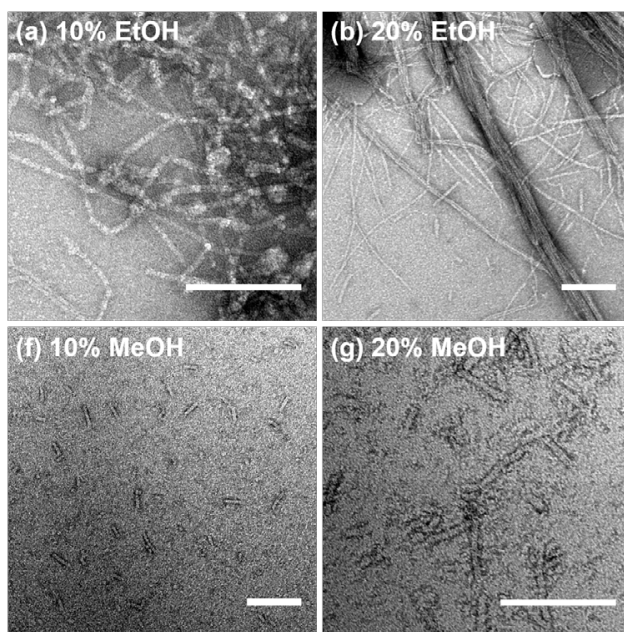
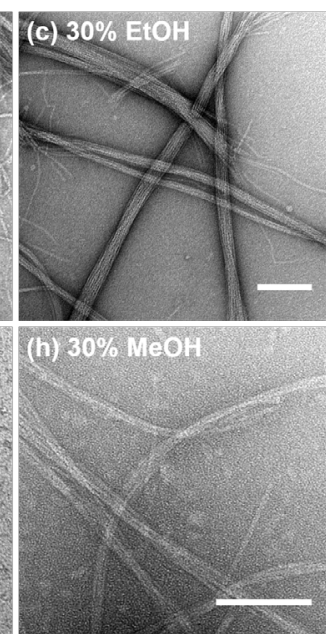
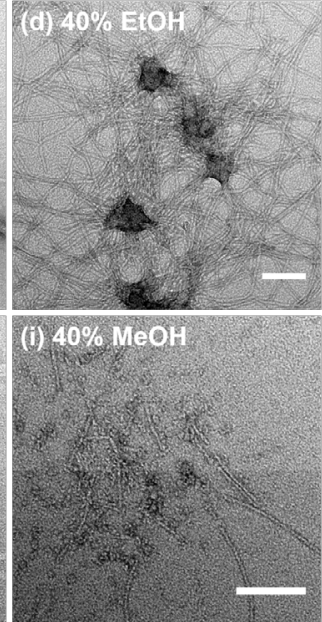
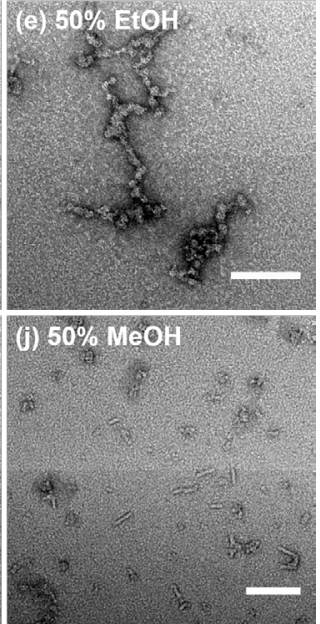

Figure 5. TEM images of nanostructures formed from $\mathrm{H} 2$ ' from analysis of solutions composed of $100 \mu \mathrm{M} \mathrm{H2}$ ', $25 \mathrm{mM}$ sodium HEPES (pH 7.4) plus varying (a-e) EtOH and (f-j) MeOH concentrations [(a, f) 10\%, (b, g) 20\%, (c, h) $30 \%,(d, i) 40 \%$ and $(e, j) 50 \%]$. Scale bars: $100 \mathrm{~nm}$.

We next sought to investigate the effect of other alcohols on the self-assembling properties of $\mathrm{H}^{\prime}$. CD-monitored titration of either methanol $(\mathrm{MeOH})$ or ethanol $(\mathrm{EtOH})$ into an aqueous solution of $\mathrm{H}^{\prime}{ }^{\prime}$ (Figure 4a-c) revealed both alcohols to increase the helicity of $\mathrm{H}^{\prime}$, warranting further study of the influence of these alcohols on $\mathrm{H} 2^{\prime}$ ' self-assembly by TEM (Figure 5). Interestingly, TEM analysis of H2' from aqueous solutions containing increasing concentrations of either $\mathrm{EtOH}$ or $\mathrm{MeOH}$ revealed the formation of fibrillar structures and a clear effect of alcohol concentration on the morphology of these structures. In the presence of EtOH (Figure 5a-e), fibrils longer than $100 \mathrm{~nm}$ were observed from solutions with $\mathrm{EtOH}$ concentrations ranging from $10 \%$ to $40 \%$. Fibril bundles were also observed from solutions with $\mathrm{EtOH}$ concentrations of $20 \%$ and $30 \%$, but disappeared at $40 \% \mathrm{EtOH}$. At an EtOH concentration of $50 \%$, amorphous aggregates only were observed. In the presence of $\mathrm{MeOH}$ (Figure $5 \mathrm{f}-\mathrm{j}$ ), protofibrils are still the dominant structure observed from solutions with $\mathrm{MeOH}$ concentrations of $10 \%$ and $20 \%$. Longitudinal growth and bundling of fibrils was observed at $30 \% \mathrm{MeOH}$, which was then attenuated as the $\mathrm{MeOH}$ concentration was increased to $40 \%$ and $50 \%$. These results indicate that the morphological evolution from protofibril to fibril bundles is consistent regardless of the type of alcohol (IPA, EtOH or MeOH) studied. However, the critical alcohol concentration for longitudinal growth of H2' fibrils (IPA (1.3 M, 10\%), EtOH (1.7 M, 10\%) and $\mathrm{MeOH}(7.4 \mathrm{M}, 30 \%)$ ) tends to decrease as the polarity of the alcohol decreases (the normalized empirical parameter of solvent polarity ( )[43] for IPA, EtOH, MeOH and water are 0.546, 0.654, 0.762 and 1.000, respectively). A similar trend was observed for amorphous aggregation (IPA (5.2 M, 40\%), EtOH (8.6 M, 50\%) and MeOH (not observed up to $12 \mathrm{M}(50 \%))$.

We then performed CD analysis to evaluate whether MRE202 is similarly affected by the polarity of the co-solvent (Figure 4c, d). The largest difference between the three co-solvents was observed at an alcohol concentration of $30 \%$, which is the optimal concentration for fibril bundle (i.e. fiber) formation. At this alcohol concentration, MRE202( $\times 10-4)$ values were found to decrease from 5.36 
(IPA), to $4.63(\mathrm{EtOH})$ and $3.81(\mathrm{MeOH})$. This decrease of MRE202 at an alcohol concentration of $30 \%$ correlates with the increase of solvent polarity. To evaluate this correlation, we plotted MRE202 versus of the alcohol-water binary solvent system[44] (Figure 4d). A quasi-linear relationship between MRE202 and is clearly shown for values above 0.8 , however, the relationship is not clear for values below 0.8 , where amorphous aggregation of $\mathrm{H}^{\prime}$ ' was observed (IPA ( $40 \%$ and $50 \%$ ) and $\mathrm{EtOH}(50 \%))$. Overall, these observations indicate that the polarity of the co-solvent is a parameter that can be used to modulate the self-assembling behaviour of $\mathrm{H2}^{\prime}$, and that self-assembly of the foldamer into long fibrils can be favoured at low alcohol concentration by decreasing the polarity of the alcohol additive (Figure 6).

\section{Kinetics of H2' fibrillogenesis}

Since the morphologies of fibrils from a single TEM sample appeared to be heterogeneous in terms of length and bundling (for example Figure $3 \mathrm{~b}$ ), we decided to follow the fibrillogenesis process of H2' over time by TEM. TEM analysis of solutions composed of $100 \mu \mathrm{M} \mathrm{H2}$ ', $25 \mathrm{mM}$ sodium HEPES ( $\mathrm{pH}$ 7.4) plus 20\% IPA at different aging times from 1 minute to 1 day (Figure 7a-e) showed that both the fibril structures and bundling are present at all time points studied. This suggests the alcoholcontrolled self-assembly to be a rapid process and the fibril structures to be stable over time. Timedependent $C D$ analysis showing negligible alteration in MRE202 upon aging time from 5 minutes to 3 days is consistent with this observation (Figure S11). Conversely, after one month of aging time in the absence of alcohols, no bundling is observed, but the short $(<100 \mathrm{~nm})$ protofibrils initially observed (i.e. after 3 days of aging time, Figure $1 e$ and $\mathrm{f}$ ) can be seen to grow longitudinally into fibrils with lengths in the micron range (Figure 7f). Overall these data suggest that the presence of IPA as a cosolvent significantly accelerates the self-assembly process, which reaches equilibrium (or full consumption of building blocks) within minutes. The acceleration of fibril formation in response to the addition of IPA is in-line with the alcohol polarity dependency described above, suggesting that both polar and hydrophobic interactions can be maximised in isopropyl alcohol/water mixtures.

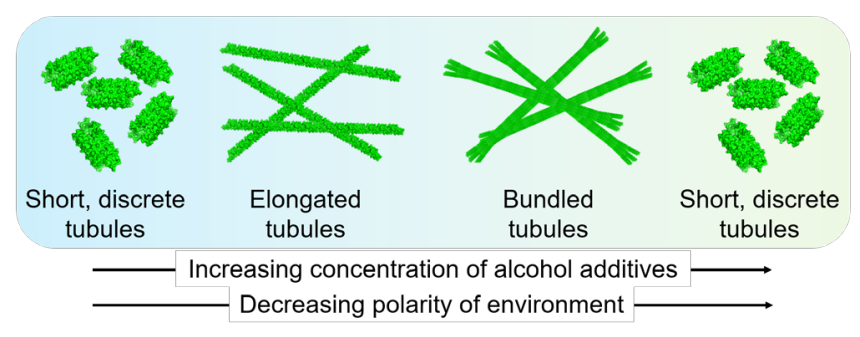

Figure 6. Schematic representation of the effect of alcohol additives and polarity on the self-assembling properties of $\mathrm{H}^{\prime}$. 

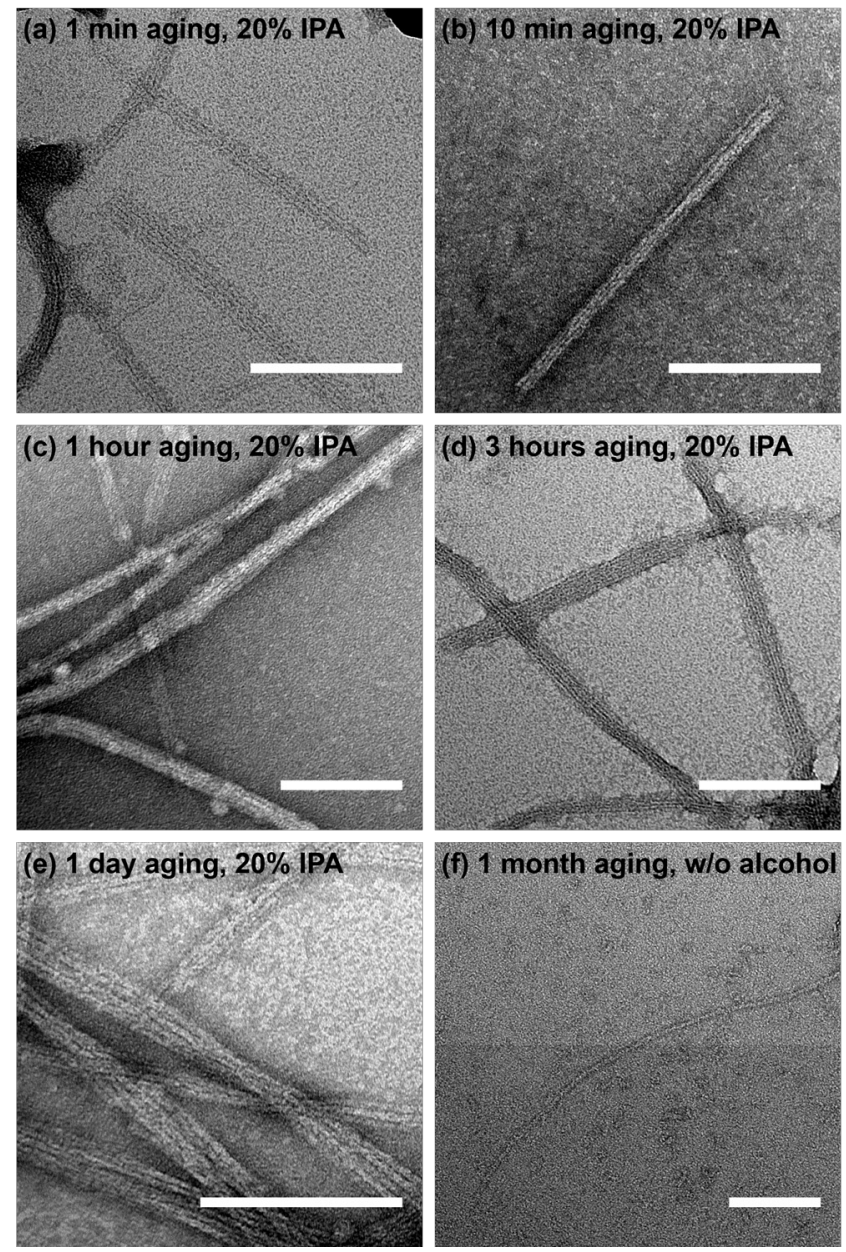

Figure 7. TEM images of $\mathrm{H}^{\prime}$ ' nanostructures (a-e) from solutions composed of $100 \mu \mathrm{M} \mathrm{H2}$ ', 25 mM sodium HEPES ( $\mathrm{pH}$ 7.4) and 20\% IPA following aging times of (a) 1 minute, (b) 10 minutes, (c) 1 hour, (d) 3 hours and (e) 1 day and ( $f$ ) in the absence of alcohol with 1 month aging time. Scale bars: $100 \mathrm{~nm}$.

\section{Conclusion}

There is considerable interest and potential in developing foldamers able to self-organise into functional assemblies with properties and functions comparable to, yet distinct from, natural biopolymers. The ability to control, or at least influence, the self-assembly process is a challenging yet necessary step towards this goal. We have shown here that an amphiphilic oligourea foldamer helix, $\mathrm{H}^{\prime}$, is able to self-assemble into a diverse array of supramolecular tubular structures. We have shown further by TEM, X-ray crystallography and CD studies that the self-assembly process, including the speed and final architecture of the assemblies, can be readily tuned by the use of alcohol additives, with both the alcohol concentration and polarity affecting the assembly process. Interestingly, the alcohol effect reported here, whereby fibrillation of $\mathrm{H}^{\prime}$ ' is maximised at a concentration of alcohol (e.g. IPA) between $20-30 \%$, parallels the behavior of a number of known natural proteins prone to form fibrils. $[37,38]$ It is hoped the work reported here will guide further investigation of oligourea foldamers as building blocks for the creation of complex and yet atomically precise nanostructures and tailored materials for potential biotechnological and biomedical applications.

\section{Experimental Section}

\section{Materials}


Unless stated otherwise, reagents were purchased from Sigma Aldrich. Distilled $\mathrm{H} 2 \mathrm{O}$ and double distilled $\mathrm{H} 2 \mathrm{O}$ were used for organic synthesis and biophysical experiments (TEM, crystallisation and $\mathrm{CD})$, respectively. Oligourea H2' was prepared by solid phase synthesis[26,45] followed by purification via reverse-phase high-performance liquid chromatography (HPLC) with its mass confirmed by electrospray ionisation mass spectrometry (ESI-MS). The full synthesis of H2' starting from Rink amide resin using appropriate azidoalkyl succinimidyl carbamate monomers is reported in the Supporting Information.

\section{Circular dichroism (CD)}

CD experiments were performed on a Jasco J-815 spectrometer. Data were recorded at $20^{\circ} \mathrm{C}$ between wavelengths of 180 and $250 \mathrm{~nm}$ at $0.5 \mathrm{~nm}$ intervals at a speed of $50 \mathrm{~nm} / \mathrm{min}$ with an integration time of 2 seconds. A quartz cell with a path length of $1 \mathrm{~mm}$ was used. H2' concentrationdependent $C D$ experiments were performed in both water and buffered conditions ( $20 \mathrm{mM}$ sodium phosphate at $\mathrm{pH}$ 7.4) starting from an $\mathrm{H}^{2}$ concentration of $200 \mu \mathrm{M}$ followed by serial two-fold dilutions. Alcohol concentration dependent $\mathrm{CD}$ experiments were performed in buffered conditions ( $20 \mathrm{mM}$ sodium phosphate at $\mathrm{pH} 7.4$ ) with a fixed $\mathrm{H}^{\prime}$ concentration as $100 \mu \mathrm{M}$.

\section{Crystallography}

For crystallisation trials, a purified lyophilized powder of $\mathrm{H} 2^{\prime}$ was dissolved in double-distilled water to a final concentration of $10 \mathrm{mg} / \mathrm{mL}$. Crystals were grown by vapour diffusion in hanging drops composed of $0.5 \mu \mathrm{L}$ of this $\mathrm{H} 2^{\prime}$ solution plus an equal volume of crystallisation reagent. Conditions suitable for crystal growth were found using standard sparse-matrix protein crystallisation screens. Two distinct crystal forms were obtained using these methods. One form, in space group P61, was obtained from crystals grown from a crystallisation reagent composed of $200 \mathrm{mM}$ sodium citrate, $100 \mathrm{mM}$ sodium HEPES buffered at pH 7.5 and $20 \%$ isopropyl alcohol. Crystals of a second crystal form (in space group P4122) were grown from a crystallisation reagent identical to that above but with an isopropyl alcohol concentration of $35 \%$. For X-ray diffraction data collection, crystals were cryo-protected in solutions composed of the relevant crystallisation reagent supplemented with $25 \%$ glycerol and flash frozen in liquid nitrogen. Diffraction data for crystals of the P61 crystal form were collected on beam line PX1 at the SOLEIL synchrotron. Diffraction data for crystals of the P4122 crystal form were collected on an FRX rotating anode home-source. Data were processed using XDS[46] (P61 crystal form), CrystalClear (P4122 crystal form) and CCP4.[47] Structures were solved by molecular replacement using Phaser[48] using Cambridge Crystallographic Data Centre entry 1030456[26] as a search model. Structures were refined in Refmac5[49] and model building performed in Coot.[50] B-factors were refined anisotropically for the P61 dataset and isotropically for the P4122 dataset. Geometric restraints for H2' were generated using PRODRG. [51] Full data collection and refinement statistics can be found in Table S1. Structures have been deposited in the Cambridge Crystallographic Data Centre database with accession codes 2010479 (P61 crystal form) and 2010480 (P4122 crystal form). These data are provided free of charge and can be accessed via http://www.ccdc.cam.ac.uk/structures.

\section{Transmission electron microscopy (TEM)}

For TEM analysis, samples were prepared in the following way: in an Eppendorf tube, sodium HEPES buffer (10 $\mu \mathrm{L}, 100 \mathrm{mM}, \mathrm{pH} 7.4)$, sodium azide solution $(4 \mu \mathrm{L}, 0.1 \%(\mathrm{w} / \mathrm{v})$ in $\mathrm{H} 2 \mathrm{O})$, alcohol (varying from $0 \mu \mathrm{L}$ to $20 \mu \mathrm{L}$ ) and $\mathrm{H} 2 \mathrm{O}$ (varying from $22 \mu \mathrm{L}$ to $2 \mu \mathrm{L}$ ) were mixed and vortexed for 5 seconds. To this solution, a solution of $\mathrm{H}^{\prime}(4 \mu \mathrm{L}, 1 \mathrm{mM}$ in $\mathrm{H} 2 \mathrm{O})$ was added to a final volume of $40 \mu \mathrm{L}$ and the tube was vortexed for 5 seconds. Unless stated otherwise, samples were incubated for 3 days before TEM analysis. For TEM analysis, $5 \mu \mathrm{L}$ of $\mathrm{H} 2$ ' solution was deposited on a carbon-coated copper grid (300 
mesh) following glow discharge (ELMO, Cordouan Technologies). After one minute, grids were dried with filter paper and stained using a $2 \%$ uranyl acetate solution. TEM images were then recorded using a FEI CM120 electron microscope at $120 \mathrm{KeV}$.

\section{Acknowledgements}

This project has received financial support from the CNRS through the MITI interdisciplinary programs (« Défi- Biomimetisme $2019 »)$ ). A post-doctoral fellowship to S.H.Yoo from IdEx Bordeaux (ANR-10-IDEX-03-02), a program of the French government managed by the Agence Nationale de la Recherche is gratefully acknowledged. We thank SOLEIL synchrotron for providing access to data collection facilities and to Pierre Legrand for assistance on beamline PX1. This work has benefited from the facilities and expertise of IECB Biophysical and Structural Chemistry platform (BPCS), CNRS UMS3033, Inserm US001, Univ. Bordeaux. We thank Marion Decossas and Oliver Lambert for their assistance with TEM studies. S.H.Y. also thanks Michael Molinari for valuable discussions. All authors thank lacovos Michaelides for critical reading of the final manuscript.

\section{Conflict of interest}

The authors declare no conflict of interest.

Keywords: Alcohols $\bullet$ Foldamers $\bullet$ Nanotubes $\bullet$ Peptidomimetics $\bullet$ Self assembly

\section{References}

[1] M. D. Shoulders, R. T. Raines, Annu. Rev. Biochem. 2009, 78, 929-958.

[2] B. J. W. Weisel, Adv. Protein Chem. 2005, 70, 247-299.

[3] J. Howard, A. A. Hyman, Nature 2003, 422, 753-758.

[4] T. Aida, E. W. Meijer, S. I. Stupp, Science 2012, 335, 813-817.

[5] Z. Luo, S. Zhang, Chem. Soc. Rev. 2012, 41, 4736-4754.

[6] G. Wei, Z. Su, N. P. Reynolds, P. Arosio, I. W. Hamley, E. Gazit, R. Mezzenga, Chem. Soc. Rev. 2017, 46, 4661-4708.

[7] D. M. Raymond, B. L. Nilsson, Chem. Soc. Rev. 2018, 47, 3659-3720.

[8] H. Cui, M. J. Webber, S. I. Stupp, Biopolymers 2010, 94, 1-18.

[9] L. Adler-Abramovich, E. Gazit, Chem. Soc. Rev. 2014, 43, 6881-6893.

[10] S. Fleming, R. V. Ulijn, Chem. Soc. Rev. 2014, 43, 8150-8177.

[11] R. J. Brea, C. Reiriz, J. R. Granja, Chem. Soc. Rev. 2010, 39, 1448-1456.

[12] C. Valéry, M. Paternostre, B. Robert, T. Gulik-Krzywicki, T. Narayanan, J. C. Dedieu, G. Keller, M. L. Torres, R. Cherif-Cheikh, P. Calvo, F. Artzner, Proc. Natl. Acad. Sci. U. S. A. 2003, 100, 1025810262.

[13] E. H. C. Bromley, K. Channon, E. Moutevelis, D. N. Woolfson, ACS Chem. Biol. 2008, 3, 38-50.

[14] T. H. Sharp, M. Bruning, J. Mantell, R. B. Sessions, A. R. Thomson, N. R. Zaccai, R. L. Brady, P. Verkade, D. N. Woolfson, Proc. Natl. Acad. Sci. U. S. A. 2012, 109, 13266-13271.

[15] H. Dong, S. E. Paramonov, J. D. Hartgerink, J. Am. Chem. Soc. 2008, 130, 13691-13695. 
[16] S. H. Gellman, Acc. Chem. Res. 1998, 31, 173-180.

[17] D. Seebach, J. Gardiner, Acc. Chem. Res. 2008, 41, 1366-1375.

[18] G. Guichard, I. Huc, Chem. Commun. 2011, 47, 5933-5941.

[19] W. S. Horne, T. N. Grossmann, Nat. Chem. 2020, 12, 331-337.

[20] D. H. Appella, L. A. Christianson, D. A. Klein, D. R. Powell, X. Huang, J. J. Barchi, S. H. Gellman, Nature 1997, 387, 381-384.

[21] V. Semetey, D. Rognan, C. Hemmerlin, R. Graff, J. P. Briand, M. Marraud, G. Guichard, Angew. Chem. Int. Ed. 2002, 41, 1893-1895.

[22] H. Jiang, J. M. Léger, I. Huc, J. Am. Chem. Soc. 2003, 125, 3448-3449.

[23] T. Sawada, S. H. Gellman, J. Am. Chem. Soc. 2011, 133, 7336-7339.

[24] F. She, P. Teng, A. Peguero-Tejada, M. Wang, N. Ma, T. Odom, M. Zhou, E. Gjonaj, L. Wojtas, A. van der Vaart, J. Cai, Angew. Chem. Int. Ed. 2018, 57, 9916-9920.

[25] P. S. P. Wang, A. Schepartz, Chem. Commun. 2016, 52, 7420-7432.

[26] G. W. Collie, K. Pulka-Ziach, C. M. Lombardo, J. Fremaux, F. Rosu, M. Decossas, L. Mauran, O. Lambert, V. Gabelica, C. D. Mackereth, G. Guichad, Nat. Chem. 2015, 7, 871-878.

[27] S. H. Yoo, H. S. Lee, Acc. Chem. Res. 2017, 50, 832-841.

[28] S. De, B. Chi, T. Granier, T. Qi, V. Maurizot, I. Huc, Nat. Chem. 2018, 10, 51-57.

[29] K. T. Nam, S. A. Shelby, P. H. Choi, A. B. Marciel, R. Chen, L. Tan, T. K. Chu, R. A. Mesch, B. C. Lee, M. D. Connolly, C. Kisielowski, R. N. Zuckermann, Nat. Mater. 2010, 9, 454-460.

[30] H. K. Murnen, A. M. Rosales, J. N. Jaworski, R. A. Segalman, R. N. Zuckermann, J. Am. Chem. Soc. 2010, 132, 16112-16119.

[31] W. C. Pomerantz, V. M. Yuwono, C. L. Pizzey, J. D. Hartgerink, N. L. Abbott, S. H. Gellman, Angew. Chem. Int. Ed. 2008, 47, 1241-1244.

[32] M. P. Del Borgo, A. I. Mechler, D. Traore, C. Forsyth, J. A. Wilce, M. C. J. Wilce, M. I. Aguilar, P. Perlmutter, Angew. Chem. Int. Ed. 2013, 52, 8266-8270.

[33] A. J. Christofferson, Z. S. Al-Garawi, N. Todorova, J. Turner, M. P. Del Borgo, L. C. Serpell, M. I. Aguilar, I. Yarovsky, ACS Nano 2018, 12, 9101-9109.

[34] P. Teng, Z. Niu, F. She, M. Zhou, P. Sang, G. M. Gray, G. Verma, L. Wojtas, A. van der Vaart, S. Ma, J. Cai, J. Am. Chem. Soc. 2018, 140, 5661-5665.

[35] M. Pasco, C. Dolain, G. Guichard, in Supramol. Chem. Water (Ed.: S. Kubik), Wiley-VCH Verlag GmbH \& Co. KGaA, Weinheim, Germany, 2019, pp. 337-374.

[36] W. Dzwolak, Biochim. Biophys. Acta - Proteins Proteomics 2006, 1764, 470-480.

[37] K. ichi Yamaguchi, H. Naiki, Y. Goto, J. Mol. Biol. 2006, 363, 279-288.

[38] S. Bucciarelli, E. S. Sayedi, S. Osella, B. Trzaskowski, K. J. Vissing, B. Vestergaard, V. Foderà, J. Colloid Interface Sci. 2020, 561, 749-761. 
[40] In the P4122 crystal form, two types of superhelices are present within the lattice with almost identical pore size and outer diameters. Details can be seen in Figure S6.

[41] E. Chovancova, A. Pavelka, P. Benes, O. Strnad, J. Brezovsky, B. Kozlikova, A. Gora, V. Sustr, M. Klvana, P. Medek, L. Biedermannova, J. Sochor, J. Damborsky, PLoS Comput. Biol. 2012, 8, e1002708.

[42] Helices of the P61 crystal form expose approximately $1900 \AA 2$ of charged surface area per helix compared to around $800 \AA 2$ of charged surface area per helix exposed in the P4122 crystal form. Surface areas were calculated using the "get_area" function in PyMOL, with a full superhelical turn analysed for each crystal form, corrected for the number of helices per turn.

[43] C. Reichardt, in Solvents Solvent Eff. Org. Chem., Wiley-VCH Verlag GmbH \& Co. KGaA, Weinheim, FRG, 2004, pp. 389-469.

[44] R. D. Skwierczynski, K. A. Connors, J. Chem. Soc. Perkin Trans. 2 1994, 4, 467-472.

[45] C. Douat-Casassus, K. Pulka, P. Claudon, G. Guichard, Org. Lett. 2012, 14, 3130-3133.

[46] W. Kabsch, Acta Cryst. 2010, D66, 125-132.

[47] M. D. Winn, C. C. Ballard, K. D. Cowtan, E. J. Dodson, P. Emsley, P. R. Evans, R. M. Keegan, E. B. Krissinel, A. G. W. Leslie, A. McCoy, S. J. McNicholas, G. N. Murshudov, N. S. Pannu, E. A. Potterton, H. R. Powell, R. J. Read, A. Vagin, K. S. Wilson, Acta Cryst. 2011, D67, 235-242.

[48] A. J. McCoy, R. W. Grosse-Kunstleve, P. D. Adams, M. D. Winn, L. C. Storoni, R. J. Read, J. Appl. Crystallogr. 2007, 40, 658-674.

[49] G. N. Murshudov, P. Skubák, A. A. Lebedev, N. S. Pannu, R. A. Steiner, R. A. Nicholls, M. D. Winn, F. Long, A. A. Vagin, Acta Cryst. 2011, D67, 355-367.

[50] P. Emsley, K. Cowtan, Acta Cryst. 2004, D60, 2126-2132.

[51] A. W. Schüttelkopf, D. M. F. Van Aalten, Acta Cryst. 2004, D60, 1355-1363. 